TRANSACTIONS OF THE

AMERICAN MATHEMATICAL SOCIETY

Volume 352, Number 1, Pages 397-412

S 0002-9947(99)02431-9

Article electronically published on July 21, 1999

\title{
VECTOR LATTICES OF WEAKLY COMPACT OPERATORS ON BANACH LATTICES
}

\author{
Z. L. CHEN AND A. W. WICKSTEAD
}

\begin{abstract}
A result of Aliprantis and Burkinshaw shows that weakly compact operators from an AL-space into a KB-space have a weakly compact modulus. Groenewegen characterised the largest class of range spaces for which this remains true whenever the domain is an AL-space and Schmidt proved a dual result. Both of these authors used vector-valued integration in their proofs. We give elementary proofs of both results and also characterise the largest class of domains for which the conclusion remains true whenever the range space is a KB-space. We conclude by studying the order structure of spaces of weakly compact operators between Banach lattices to prove results analogous to earlier results of one of the authors for spaces of compact operators.
\end{abstract}

\section{INTRODUCTION}

In 1981 Aliprantis and Burkinshaw [8] proved that every weakly compact operator from an AL-space into a KB-space has a weakly compact modulus. Proofs of this may be found in, for example, Theorem 3.5.14 of [14] or Theorem 17.14 of [9]. In 1982, G. Groenewegen [12] extended this result to the case of operators from an AL-space into a Banach lattice with what he termed property (W1) (see below for the definition), by representing operators by means of vector-valued integration. He also showed that this was the widest possible extension of the class of range spaces for which such a result held. Here we will provide an elementary proof of this result using techniques similar to those used in [17] when dealing with compact operators. We will also prove a dual version of this result which generalizes a result proved in 1988 by Schmidt [16]. We further extend Aliprantis and Burkinshaw's result by finding the widest possible class of domains $E$ for which each regular weakly compact operator from $E$ into a KB-space has a weakly compact modulus.

Recently in [6] it has been shown that there exist a Dedekind complete Banach lattice $E$ and a compact operator $T$ on $E$ dominated by a positive compact operator such that the modulus $|T|$ of $T$ is not compact. It is natural to consider the corresponding problem for weakly compact operators. In the spirit of [6] we give an example which shows the existence of a Dedekind complete Banach lattice $E$ and a compactly dominated compact operator $T$ on $E$ such that the modulus $|T|$ of $T$ is not weakly compact.

In [17] the order properties of some spaces of compact operators between two Banach lattices was considered. In $\S 3$ we will prove analogous results for weakly

Received by the editors May 7, 1997.

1991 Mathematics Subject Classification. Primary 47B65; Secondary 47 B07.

Key words and phrases. Weakly compact operators, Banach lattices.

(C)1999 American Mathematical Society 
compact operators. In particular we show precisely when, assuming that either $E$ is an AL-space or $F$ an AM-space, the space of all weakly compact operators from $E$ into $F$ is a vector lattice and, in a completely general setting, when the linear span of the positive weakly compact operators forms a Dedekind complete vector lattice. In several places in this paper we point out where similar results for Dunford-Pettis operators also hold.

If $E$ and $F$ are Banach lattices, then $\mathcal{L}(E, F)$ and $\mathcal{K}(E, F)$ will respectively denote the spaces of all bounded and of all compact operators from $E$ into $F$. The space of regular operators from $E$ into $F$ is denoted by $\mathcal{L}^{r}(E, F)$, and $\mathcal{K}^{r}(E, F)$ denotes the linear span of the positive compact operators from $E$ into $F$. For general Banach lattice terminology we follow [9] or [14].

We reserve the notation $e_{n}$ to denote the sequence of reals with all terms zero except for the $n$ 'th which is 1 , and $\mathbf{1}$ for the constantly 1 sequence.

\section{THE MOdULI OF WEAKLY COMPACT OPERATORS}

The following definition was introduced by Groenewegen in [12].

Definition 2.1. A Banach lattice $\mathrm{E}$ is said to have property (W1) if for each relatively weakly compact subset $\mathrm{A}$ of $\mathrm{E},|A|=\{|x|: x \in A\}$ is also relatively weakly compact.

Although this property first seems to have been mentioned by Groenewegen, there are antecedents in the literature. Abramovich in [1] considered a very similar condition involving only disjoint sets. Also Groenewegen defines property (W3) to be that the solid hull of every weakly compact set is again weakly compact. This was also introduced much earlier by Abramovich in [1] under the name of condition $(N)$. The same paper also seems to have been the first to use generalized Rademacher functions in an abstract Banach lattice setting as well as containing precursors of many results subsequently published independently in the western literature.

It is proved in [1], Theorems 3.2 and 3.3 (see [9], Theorem 13.8 for a more accessible proof), that each KB-space has property (W3) and hence has property (W1) and it is clear that if the lattice operations of a Banach lattice $E$ are weakly sequentially continuous, then $E$ has property (W1). In particular, each AM-space has property (W1) by Proposition 2.1.11 of [14]. In [10] we characterised such Banach lattices which also had an order continuous norm as those which were an order direct sum of a KB-space and an atomic Banach lattice with an order continuous norm. Thus $c_{0}\left(L_{1}[0,1]\right)$ provides an example of a Banach lattice with an order continuous norm which does not have property (W1). It is simple to provide a self-contained proof of this fact, so we present that here for completeness.

Example 2.2. Let $E=c_{0}\left(L_{1}[0,1]\right)$, then the norm of $E$ is order continuous but $E$ does not have property (W1).

Proof. Indeed, let $r_{n}(t)=\operatorname{sgn}\left(\sin 2^{n} \pi t\right)$, the $n$ 'th Rademacher functions on $[0,1]$, then $r_{n}(t) \rightarrow 0$ weakly in $L_{1}[0,1]$ as $n \rightarrow \infty$. Define

$$
x_{n}=(\overbrace{r_{n}, \ldots, r_{n}}^{n}, 0, \ldots) \in E
$$


then it is not difficult to verify that $x_{n} \rightarrow 0$ weakly in $E$ as $n \rightarrow \infty$, and that

$$
\left|x_{n}\right|=(\overbrace{\left|r_{n}\right|, \ldots,\left|r_{n}\right|}^{n}, 0, \ldots)=(\overbrace{\chi_{[0,1]}, \ldots, \chi_{[0,1]}}^{n}, 0, \ldots)
$$

is increasing with $\left\|\left|x_{n}\right|-\left|x_{m}\right|\right\|=1$ if $n \neq m$. Thus the sequence $\left(\left|x_{n}\right|\right)$ does not contain any weakly convergent subsequence, and $E$ fails to have property (W1).

The following result has, essentially, the same statement as Theorem V.3.8 of [12], but the proof is very different. In particular we avoid the use of vector-valued integration. For that reason, plus the relative inaccessibility of [12], we include a complete proof here.

Theorem 2.3. For a Banach lattice $F$ the following assertions are equivalent:

(1) F has property (W1).

(2) For each AL-space E, every weakly compact operator from $E$ into $F$ has a weakly compact modulus.

(3) Every weakly compact operator from $\ell_{1}$ into $F$ has a weakly compact modulus.

Proof. (1) $\Rightarrow(2)$. Suppose $F$ has property (W1), $E$ is an AL-space and $T: E \rightarrow F$ is a weakly compact operator. We will show the modulus $|T|$ of $T$ exists and is weakly compact. Let $E_{1}$ denote the unit ball in $E$ and let $E_{1+}$ denote its positive part. $A=T E_{1+}$ is relatively weakly compact and by (1) so is $|A|$. It follows from the Krein-Smulyan theorem that the closed convex hull $K$ of $|A|$ is weakly compact.

For each $x \in E_{1+}$, put

$$
A_{x}=\left\{\sum_{i=1}^{n}\left|T x_{i}\right|: n \in \mathbb{N}, x_{i} \geq 0, \sum_{i=1}^{n} x_{i}=x\right\}
$$

then $A_{x}$ is upward directed. Moreover $A_{x} \subset K$. In fact,

$$
\sum_{i=1}^{n}\left|T x_{i}\right|=\sum_{i=1}^{n}\left\|x_{i}\right\|\left|T\left(x_{i} /\left\|x_{i}\right\|\right)\right|=\sum_{i=1}^{n}\left\|x_{i}\right\|\left|y_{i}\right|
$$

where $y_{i}=T\left(x_{i} /\left\|x_{i}\right\|\right) \in A$, and $\sum_{i=1}^{n}\left\|x_{i}\right\|=\left\|\sum_{i=1}^{n} x_{i}\right\|=\|x\| \leq 1$ since $E$ is an AL-space. Noting that $0 \in K$, it follows that $\sum_{i=1}^{n}\left|T x_{i}\right| \in K$, i.e. $A_{x} \subset K$.

Being an upward directed subset of a weakly compact set $K, A_{x}$ weakly converges, and hence norm converges, to its supremum, $y(x) \in K$. Now Theorem 1.10 of [9] shows that the modulus $|T|$ of $T$ exists, and that $|T| x=y(x) \in K$ for all $x \in E_{1+}$. It follows that $|T|\left(E_{1}\right)$ is relatively weakly compact so that $|T|$ is weakly compact. Also let us take this opportunity to point out that

$$
\|T\|_{r}=\||T|\|=\|T\| .
$$

Indeed

$$
\||T| x\|=\|y(x)\|=\lim \left\{\|y\|: y \in A_{x}\right\} \leq\|T\|
$$


for each $x \in E_{1+}$, as

$$
\begin{aligned}
\left\|\sum_{i=1}^{n}\left|T x_{i}\right|\right\| & =\left\|\sum_{i=1}^{n}\right\| x_{i}\left\|\mid T\left(x_{i} /\left\|x_{i}\right\|\right)\right\| \\
& \leq \sum_{i=1}^{n}\left\|x_{i}\right\|\left\|T\left(x_{i} /\left\|x_{i}\right\|\right)\right\| \\
& \leq\|T\|\left\|\sum_{i=1}^{n} x_{i}\right\| \\
& \leq\|T\| .
\end{aligned}
$$

It is clear that $(2) \Rightarrow(3)$. Now we show that $(3) \Rightarrow(1)$. Assuming that (3) holds but that (1) is false, there exists a weakly convergent sequence $x_{n} \in F$ such that $\left\{\left|x_{n}\right|\right\}_{n=1}^{\infty}$ does not contain any weakly convergent subsequence. Define $T: \ell_{1} \longrightarrow F$ by $T\left(\lambda_{i}\right)=\sum_{i=1}^{\infty} \lambda_{i} x_{i}$ for $\left(\lambda_{i}\right) \in \ell_{1}$, then Corollary 10.16 of [9] implies that $T$ is weakly compact. By (3) $T$ has a weakly compact modulus. It is easy to verify that, in fact,

$$
|T|\left(\lambda_{i}\right)=\sum_{i=1}^{\infty} \lambda_{i}\left|x_{i}\right|
$$

for all $\left(\lambda_{i}\right) \in \ell_{1}$, so the weak compactness of $|T|$ implies that $\left\{\left|x_{i}\right|=|T| e_{i}\right\}_{i=1}^{\infty}$ contains a weakly convergent subsequence, which is a contradiction, establishing that $(3) \Rightarrow(1)$.

The following result is dual to Theorem 2.3 and generalises the result of Schmidt in [16] in the same way that Theorem 2.3 generalises the result of Aliprantis and Burkinshaw.

Theorem 2.4. For a Banach lattice $E$ the following assertions are equivalent:

(1) $E^{\prime}$ has property (W1).

(2) For each Dedekind complete AM-space F with unit, every weakly compact operator from $E$ into $F$ has a weakly compact modulus.

(3) E very weakly compact operator from $E$ into $\ell_{\infty}$ has a weakly compact modulus.

Proof. $(1) \Rightarrow(2)$. Since $F$ is a Dedekind complete AM-space with unit, by Proposition 1.5.10 in [14] there exists a positive contractive projection $P: F^{\prime \prime} \rightarrow F$. Proposition 1.5.12 of [14] implies that

$$
|T|=P\left|T^{\prime}\right|^{\prime} j
$$

for all $T \in \mathcal{L}^{r}(E, F)=\mathcal{L}(E, F)$, where $j: E \rightarrow E^{\prime \prime}$ is the natural inclusion mapping. If $T$ is weakly compact, so is $T^{\prime}$ by Gantmacher's theorem. Note that $T^{\prime}: F^{\prime} \rightarrow$ $E^{\prime}, F^{\prime}$ is an AL-space by Theorem 12.22 of [9], thus it follows from Theorem 2.3 above that $\left|T^{\prime}\right|$ is weakly compact and hence, by Gantmacher's theorem, so is $\left|T^{\prime}\right|^{\prime}$ and hence so is $|T|=P\left|T^{\prime}\right|^{\prime} j$.

It is clear that $(2) \Rightarrow(3)$. To prove that $(3) \Rightarrow(1)$, assume that $E^{\prime}$ does not have property (W1), then there exists a weakly convergent sequence $x_{n}^{\prime} \in E^{\prime}$, say $x_{n}^{\prime} \rightarrow x_{0}^{\prime} \in E^{\prime}$ weakly as $n \rightarrow \infty$, but with $\left\{\left|x_{n}^{\prime}\right|\right\}_{n=1}^{\infty}$ containing no weakly convergent subsequence. 
Define $T: E \rightarrow \ell_{\infty}$ by $T x=\left(x_{n}^{\prime}(x)\right)_{n=1}^{\infty}$, then $T$ is weakly compact. Indeed, if we define $S: E \rightarrow c_{0} \subset \ell_{\infty}$ by $S x=\left(x_{n}^{\prime}(x)-x_{0}^{\prime}(x)\right)_{n=1}^{\infty}$ and $S_{0}: E \rightarrow \ell_{\infty}$ by $S_{0}=x_{0}^{\prime}(x) e_{0}$, where $e_{0}=(1,1, \ldots) \in \ell_{\infty}$, then obviously $T=S+S_{0}$ and $S_{0}$ is of rank 1, whilst from Theorem 17.5 of [9] $S$ is weakly compact as an operator into $c_{0}$ and hence into $\ell_{\infty}$. Hence $T$ is weakly compact. By (3) $|T|$ is weakly compact, and it is easy to see that $|T| x=\left(\left|x_{n}^{\prime}\right|(x)\right)_{n=1}^{\infty}$ for all $x \in E$, so that

$$
|T|^{\prime}\left(\lambda_{n}\right)=\sum_{n=1}^{\infty} \lambda_{n}\left|x_{n}^{\prime}\right|
$$

for all $\left(\lambda_{n}\right) \in \ell_{1} \subset\left(\ell_{\infty}\right)^{\prime}$. Gantmacher's theorem implies that $|T|^{\prime}$ is weakly compact, which shows that $\left\{\left|x_{n}^{\prime}\right|: n \in \mathbb{N}\right\}=\left\{|T|^{\prime} e_{n}: n \in \mathbb{N}\right\}$ contains a weakly convergent subsequence, where $e_{n} \in \ell_{1}$ denotes the sequence with $n$ 'th entry equal to 1 and all others zero. This is a contradiction, thus $(3) \Rightarrow(1)$ holds.

We now give an example to show that if we only suppose that $F$ is a Dedekind complete AM-space (without unit), the results in Theorem 2.4 are false even if $E$ is an AM-space.

Example 2.5. Let $E=C([0,1]), F=\ell_{\infty}\left(F_{n}\right)$ where $F_{n}=\left(\ell_{\infty},\|\cdot\|\right)$ and $\left\|\left(\lambda_{k}\right)\right\|=$ $\max \left\{\left\|\left(\lambda_{k}\right)\right\|_{\infty}, n \lim \sup \left(\left|\lambda_{k}\right|\right)\right\}$ for all $\left(\lambda_{k}\right) \in \ell_{\infty}$. Then for each $n \in \mathbb{N}, F_{n}$ is a Dedekind complete AM-space, hence so is $F$.

Define $T_{n}: E \rightarrow F_{n}$ by $T_{n} f=\left(2^{n} \int_{I_{n}} f r_{k} d t\right)_{k=1}^{\infty} \in F_{n}$ for all $f \in E$, where $r_{n}$ is the $n$ 'th Rademacher function on $[0,1]$ and $I_{n}=\left(2^{-n}, 2^{-n+1}\right)$. Since $r_{n} \rightarrow 0$ weakly in $L_{1}(0,1)$ as $n \rightarrow \infty$, and $L_{1}[0,1]$ is a closed subspace of $E^{\prime}$, it follows that $r_{n} \rightarrow 0$ weakly in $E^{\prime}$ as $n \rightarrow \infty$. Also $T_{n}: E \rightarrow c_{0} \subset F_{n}$, so that Theorem 17.5 of [9] implies that $T_{n}$ is weakly compact for all $n \in \mathbb{N}$. (Note that $r_{k} \chi_{I_{n}} \rightarrow 0$ weakly in $E^{\prime}$ as $k \rightarrow \infty$.)

On the other hand, for each $f \in E$ and each $n \in \mathbb{N}$

$$
\left\|T_{n} f\right\|_{F_{n}}=\left\|\left(2^{n} \int_{I_{n}} f r_{k} d t\right)_{k=1}^{\infty}\right\|_{\infty} \leq\|f\|
$$

so that $\|T\| \leq 1$. It is easy to check that the modulus $\left|T_{n}\right|$ of $T_{n}$ exists and that $\left|T_{n}\right| f=\left(2^{n} \int_{I_{n}} f d t\right) e_{0} \in F_{n}$, where $e_{0}=(1,1, \ldots)$, so that $\left\|T_{n}\right\|_{r}=\left\|\left|T_{n}\right|\right\|=n$ for all $n \in \mathbb{N}$.

Now we define $T: E \rightarrow F$ by $T f=\left(\frac{1}{n} T_{n} f\right)_{n=1}^{\infty}$, then $T$ is weakly compact as the limit of weakly compact operators, and its modulus $|T|$ exists and satisfies

$$
|T| f=\left(\frac{1}{n}\left|T_{n}\right| f\right)_{n=1}^{\infty}=\left(\left(2^{n} \int_{I_{n}} f d t\right) \frac{e_{0}}{n}\right)_{n=1}^{\infty}=\left(\left(2^{n} \int_{I_{n}} f d t\right) \bar{e}_{n}\right)_{n=1}^{\infty}
$$

where $\bar{e}_{n}=\frac{1}{n} e_{0} \in F_{n}$ so that $\left\|\bar{e}_{n}\right\|_{F_{n}}=1$ for all $n \in \mathbb{N}$.

We claim that $|T|$ is not weakly compact. In fact, choose $f_{n} \in E$ with $0 \leq f_{n} \leq$ $1, f_{n}(t)=0$ if $t \notin I_{n}$ and $\int_{I_{n}} f_{n} d t=2^{-n-1}$. The $f_{n}$ are order bounded and disjoint and

$$
\left\||T| f_{n}\right\| \geq\left\|\frac{1}{n}\left|T_{n}\right| f_{n}\right\|=2^{n} \int_{I_{n}} f_{n} d t=\frac{1}{2}
$$

for all $n \in \mathbb{N}$. Therefore Theorem 3.4.4 of [14] implies that $|T|$ is not order-weakly compact and hence not weakly compact.

Now let us turn our attention to the following problem: What happens if we have a weaker condition on $E$ and a stronger one on $F$ in Theorem 2.3? For example if 
we assume that $F$ is a KB-space, for what $E$ is the result in Theorem 2.3 still true? To this end let us recall from [13] that a Banach lattice $E$ is order bounded $A M$ if whenever $x_{n} \in E$ is an unconditionally summable sequence such that $\left\{\sum_{i=1}^{n} x_{i}\right\}_{n=1}^{\infty}$ is order bounded, then $\left\{\left|x_{n}\right|\right\}_{n=1}^{\infty}$ is summable. Every AM-space is order bounded $\mathrm{AM}$, as is every Banach lattice with an order continuous norm; see [13] for details. This terminology may seem to be a rather strange one. One justification for it is that if the order boundedness condition is dropped and one merely asks that every unconditionally summable sequence be absolutely summable, then Abramovich, Posizelsky and Janovsky showed in [2], in response to a question of G. Jameson, that an isomorphic characterisation of AM-spaces is obtained.

Theorem 2.6. For a Banach lattice $E$ the following assertions are equivalent:

(1) $E^{\prime}$ is order bounded $A M$.

(2) For each $K B$-space $F$, every regular weakly compact operator from $E$ into $F$ has a weakly compact modulus.

(3) Every weakly compact operator from $E$ into $\ell_{1}$ has a weakly compact modulus.

Proof. (1) $\Rightarrow(2)$. Let $F$ be a KB-space and $T: E \rightarrow F$ be a regular weakly compact operator, so that $|T|$ exists. If $|T|$ is not weakly compact, then by Theorem 1.4 of $[15]$ there exists an order bounded sequence of pairwise disjoint positive elements $x_{n}^{\prime} \in F^{\prime}$ such that

$$
\sup \left\{\left|x_{n}^{\prime}(|T| x)\right|: x \in \operatorname{ball}(E)\right\}=\left\||T|^{\prime} x_{n}^{\prime}\right\| \geq \epsilon>0
$$

for some $\epsilon>0$ and all $n \in \mathbb{N}$. According to Theorem 5.11 of $[9],\left|T^{\prime}\right|=|T|^{\prime}$, so $\left\|\left|T^{\prime}\right| x_{n}^{\prime}\right\| \geq \epsilon>0$ for all $n \in \mathbb{N}$.

For each $n$ apply Theorem 1.16 of [9] (remembering that the norm of $E^{\prime}$ is Fatou) to see that there exist $m_{n} \in \mathbb{N}, z_{n 1}^{\prime}, \ldots, z_{n m_{n}}^{\prime} \in F_{+}^{\prime}$ such that

$$
\sum_{i=1}^{m_{n}} z_{n i}^{\prime}=x_{n}^{\prime}
$$

$$
\left\|\sum_{i=1}^{m_{n}}\left|T^{\prime} z_{n i}^{\prime}\right|\right\|>\frac{1}{2} \epsilon
$$

Choose $x^{\prime} \in E^{\prime}$ such that $\sum_{i=1}^{n} x_{i}^{\prime} \leq x^{\prime}$ for all $n \in \mathbb{N}$. It is clear that the sequence of partial sums of the series

$$
z_{11}^{\prime}+\cdots+z_{1 m_{1}}^{\prime}+z_{21}^{\prime}+\cdots+z_{2 m_{2}}^{\prime}+z_{31}^{\prime}+\cdots
$$

is increasing and order bounded, so that it is a weak Cauchy sequence. Since $T$ is weakly compact, so is $T^{\prime}$ by Gantmacher's theorem, and it follows that the partial sums of the series $(*)$

$$
T^{\prime} z_{11}^{\prime}+\cdots+T^{\prime} z_{1 m_{1}}^{\prime}+T^{\prime} z_{21}^{\prime}+\cdots+T^{\prime} z_{2 m_{2}}^{\prime}+T^{\prime} z_{31}^{\prime}+\cdots
$$

form a weakly convergent sequence. For the same reason we know that each subseries of $(*)$ is also weakly convergent. Thus the Orliz-Pettis Theorem (see [11], Chapter IV) implies that the sequence

$$
T^{\prime} z_{11}^{\prime}, \ldots, T^{\prime} z_{1 m_{1}}^{\prime}, T^{\prime} z_{21}^{\prime}, \ldots, T^{\prime} z_{2 m_{2}}^{\prime}, T^{\prime} z_{31}^{\prime}, \ldots
$$


is unconditionally summable. Also for each $n \in \mathbb{N}$,

$$
\sum_{i=1}^{n} \sum_{j=1}^{m_{i}}\left|T^{\prime} z_{i j}^{\prime}\right| \leq \sum_{i=1}^{n} \sum_{j=1}^{m_{i}}\left|T^{\prime}\right| z_{i j}^{\prime}=\left|T^{\prime}\right|\left(\sum_{i=1}^{n} x_{i}^{\prime}\right) \leq\left|T^{\prime}\right| x^{\prime}
$$

so that the sequence

$$
\left|T^{\prime} z_{11}^{\prime}\right|, \ldots,\left|T^{\prime} z_{1 m_{1}}^{\prime}\right|,\left|T^{\prime} z_{21}^{\prime}\right|, \ldots,\left|T^{\prime} z_{2 m_{2}}^{\prime}\right|,\left|T^{\prime} z_{31}^{\prime}\right|, \ldots
$$

has order bounded partial sums. The latter sequence is summable, as $E^{\prime}$ is order bounded AM, so that

$$
\left\|\sum_{i=1}^{m_{n}}\left|T^{\prime} z_{n i}^{\prime}\right|\right\| \rightarrow 0 \quad \text { as } \quad n \rightarrow \infty
$$

which contradicts (b) above. Therefore $|T|$ is weakly compact.

Obviously $(2) \Rightarrow(3)$. Now assume (3) and that $\left\{x_{n}^{\prime}\right\}_{n=1}^{\infty}$ is an unconditionally summable sequence in $E^{\prime}$ such that

$$
\sum_{i=1}^{n}\left|x_{i}^{\prime}\right| \leq x^{\prime} \quad \forall n \in \mathbb{N} .
$$

Then for each $x \in E$, and each $x^{\prime \prime} \in E^{\prime \prime}$, we have

$$
\begin{gathered}
\sum_{i=1}^{n}\left|x_{i}^{\prime}(x)\right| \leq \sum_{i=1}^{n}\left|x_{i}^{\prime}\right|(|x|) \leq x^{\prime}(x), \\
\sum_{i=1}^{n}\left|x^{\prime \prime}\left(x_{i}^{\prime}\right)\right| \leq \sum_{i=1}^{n}\left|x^{\prime \prime}\right|\left(\left|x_{i}^{\prime}\right|\right) \leq\left|x^{\prime \prime}\right|\left(\left|x^{\prime}\right|\right)
\end{gathered}
$$

for all $n \in \mathbb{N}$, so that $\left(x_{n}^{\prime}(x)\right),\left(\left|x_{n}^{\prime}\right|(x)\right),\left(x^{\prime \prime}\left(x_{n}^{\prime}\right)\right) \in \ell_{1}$ for all $x \in E, x^{\prime \prime} \in E^{\prime \prime}$.

Now define $T: E \rightarrow \ell_{1}$ by $T x=\left(x_{n}^{\prime}(x)\right)$ for $x \in E$. It is easy to verify that $T \in \mathcal{L}^{r}\left(E, \ell_{1}\right)$ and that the modulus $|T|$ of $T$ exists with

$$
|T| x=\left(\left|x_{n}^{\prime}\right|(x)\right), \quad x \in E .
$$

Also $T^{\prime}: \ell_{\infty} \rightarrow E^{\prime}$ has $T^{\prime}\left(\lambda_{n}\right)=\sum_{n=1}^{\infty} \lambda_{n} x_{n}^{\prime}$, for $\left(\lambda_{n}\right) \in \ell_{\infty}$, which series is norm convergent as $\left\{x_{n}^{\prime}\right\}_{n=1}^{\infty}$ is unconditionally summable. Then $T^{\prime \prime}: E^{\prime \prime} \rightarrow\left(\ell_{1}\right)^{\prime \prime}=$ $\left(\ell_{\infty}\right)^{\prime}$, and for each $x^{\prime \prime} \in E^{\prime \prime}$ and $\left(\lambda_{n}\right) \in \ell_{\infty}$ we have

$$
T^{\prime \prime} x^{\prime \prime}\left(\left(\lambda_{n}\right)\right)=x^{\prime \prime}\left(T^{\prime}\left(\lambda_{n}\right)\right)=\sum_{n=1}^{\infty} \lambda_{n} x^{\prime \prime}\left(x_{n}^{\prime}\right)
$$

and it follows that

$$
T^{\prime \prime} x^{\prime \prime}=\left(x^{\prime \prime}\left(x_{n}^{\prime}\right)\right) \in \ell_{1} \quad \forall x^{\prime \prime} \in E^{\prime \prime},
$$

which tells us that $T^{\prime \prime} E^{\prime \prime} \subset \ell_{1}$, so that Theorem 17.2 of [9] implies that $T$ is weakly compact, and by (3) $|T|$ also is weakly compact, as is $|T|^{\prime}$ by Gantmacher's theorem. Note that

$$
\left(|T|^{\prime} e_{n}\right)(x)=e_{n}(|T| x)=\left|x_{n}^{\prime}\right|(x)
$$

for all $x \in E$, so that $|T|^{\prime} e_{n}=\left|x_{n}^{\prime}\right|$ for each $n \in \mathbb{N}$. Since $u_{n}=e_{1}+e_{2}+\cdots+e_{n}$ is a weak Cauchy sequence in $\ell_{\infty}$, the weak compactness of $|T|^{\prime}$ implies that the increasing sequence $\left(\left|x_{1}^{\prime}\right|+\left|x_{2}^{\prime}\right|+\cdots+\left|x_{n}^{\prime}\right|\right)=\left(|T|^{\prime} u_{n}\right)$ is weakly convergent, and hence norm convergent. This means that $\left\{\left|x_{n}^{\prime}\right|\right\}_{n=1}^{\infty}$ is summable, so that $E^{\prime}$ is order bounded AM and hence $(3) \Rightarrow(1)$ holds. 
In $[6]$ it was shown that it is possible for a compact operator on a Dedekind complete Banach lattice, which is dominated by a positive compact operator, to have a modulus which is not compact. Our next result shows that in these circumstances the modulus need not even be weakly compact.

Theorem 2.7. (1) There exist Dedekind complete Banach lattices $E$ and $F$ and compact operators $S, T: E \rightarrow F$ with $S,-S \leq T$ but with the modulus $|S|$ of $S$ not weakly compact.

(2) There exist a Dedekind complete Banach lattice $E$ and compact operators $S$, $T$ on $E$ with $S,-S \leq T$ such that the modulus $|S|$ of $S$ is not weakly compact.

Proof. Let us recall from [6] that $S_{n}$ is an operator on $2^{n}$-dimensional Euclidean space $\ell_{2}^{2^{n}}$ with $\left\|S_{n}\right\|=1$ and $\left\|\left|S_{n}\right|\right\|=2^{n}$. The operator $J_{n}$ embeds $\ell_{2}^{2^{n}}$ into $L_{2}[0,1]$ by the formula

$$
J_{n}\left(x_{1}, x_{2}, \ldots, x_{2^{n}}\right)=\sum_{k=1}^{2^{n}} x_{k} \chi_{\left[(k-1) 2^{-n}, k 2^{-n}\right]} .
$$

Recall also that for each $n \in \mathbb{N}$ we have $\left\|J_{n} S_{n}\right\|=1,\left\|J_{n}\left|S_{n}\right|\right\|=2^{n / 2}$ and that $\left|J_{n} S_{n}\right|\left(x_{k}\right)=J_{n}\left|S_{n}\right|\left(x_{k}\right)=\left(\sum_{k=1}^{2^{n}} x_{k}\right) \chi_{[0,1]}=2^{n / 2}\left(x_{k}\right)\left(v_{n}\right) \chi_{[0,1]}$, where $v_{n}=$ $2^{-n / 2}(1,1, \ldots, 1) \in \ell_{2}^{2^{n}}$, so that $\left\|v_{n}\right\|_{2}=1$.

Let $E=\ell_{1}\left(\ell_{2}^{2^{n}}\right)$ and $F=\ell_{\infty}\left(L_{2}[0,1]\right)$ so that both $E$ and $F$ are Dedekind complete. Define $S, T: E \rightarrow F$ by

$$
S \mathbf{x}=\left(\sum_{k=n}^{\infty} 2^{-k / 2} J_{k} S_{k} \mathbf{x}_{k}\right)_{n=1}^{\infty},
$$

and

$$
\begin{aligned}
T \mathbf{x} & =\left(\sum_{k=1}^{\infty} 2^{-k / 2} J_{k}\left|S_{k}\right| \mathbf{x}_{k}\right)_{n=1}^{\infty} \\
& =\left(\sum_{k=1}^{\infty} \mathbf{x}_{k}\left(v_{k}\right) \chi_{[0,1]}\right)_{n=1}^{\infty}
\end{aligned}
$$

for $\mathbf{x}=\left(\mathbf{x}_{n}\right) \in E$.

Clearly $T$ is defined and compact as it is bounded and of rank 1 and it is also clear that $S,-S \leq T$. The operator $S$ is also compact. Indeed, for each $n \in \mathbb{N}$ define $H_{n}: E \rightarrow L_{2}[0,1]$ by

$$
H_{n} \mathbf{x}=\sum_{k=n}^{\infty} 2^{-k / 2} J_{k} S_{k} \mathbf{x}_{k}
$$

and $H_{n m}: E \rightarrow L_{2}[0,1]$ by

$$
H_{n m}(\mathbf{x})=\sum_{k=n}^{n+m} 2^{-k / 2} J_{k} S_{k} \mathbf{x}_{k}
$$

then all $H_{n m}$ are compact, as they are finite rank,

$$
\left\|H_{n}-H_{n m}\right\| \leq \sum_{k=n+m+1}^{\infty} 2^{-k / 2} \rightarrow 0 \quad \text { as } \quad m \rightarrow \infty,
$$

so each $H_{n}$ is compact. 
VECTOR LATTICES OF WEAKLY COMPACT OPERATORS ON BANACH LATTICES 405

Let $U_{n}: E \rightarrow F$ by $U_{n}(\mathbf{x})=\left(H_{1}\left(\mathbf{x}_{k}\right), \ldots, H_{n}\left(\mathbf{x}_{k}\right), 0, \ldots\right)$ for $\mathbf{x}=\left(\mathbf{x}_{k}\right) \in E$. Then $U_{n}$ is compact and

$$
\left\|S-U_{n}\right\| \leq \sum_{k=n+1}^{\infty} 2^{-k / 2}\left\|J_{k} S_{k}\right\|=\sum_{k=n+1}^{\infty} 2^{-k / 2} \rightarrow 0 \quad \text { as } n \rightarrow \infty
$$

so it follows that $S$ is compact.

Now we claim that the modulus $|S|$ of $S$ is not weakly compact. In fact, it is easy to check that

$$
|S| \mathbf{x}=\left(\sum_{k=n}^{\infty} 2^{-k / 2} J_{k}\left|S_{k}\right| \mathbf{x}_{k}\right)_{n=1}^{\infty}=\left(\left(\sum_{k=n}^{\infty} \mathbf{x}_{k}\left(v_{k}\right)\right) \chi_{[0,1]}\right)
$$

for all $\mathbf{x}=\left(\mathbf{x}_{k}\right) \in E$. Let $\mathbf{v}_{n}$ denote the sequence with $n$ 'th entry equal to $v_{n}$ and all others zero, so that $\left\|\mathbf{v}_{n}\right\|=1$ and

$$
\begin{aligned}
|S| \mathbf{v}_{n} & =(\overbrace{v_{n}\left(v_{n}\right) \chi_{[0,1]}, \ldots, v_{n}\left(v_{n}\right) \chi_{[0,1]}}^{n}, 0, \ldots) \\
& =(\overbrace{\chi_{[0,1]}, \ldots, \chi_{[0,1]}}^{n}, 0, \ldots) .
\end{aligned}
$$

Clearly $|S| \mathbf{v}_{n} \uparrow$ and $\left\||S| \mathbf{v}_{n}-|S| \mathbf{v}_{m}\right\|=1$ if $n \neq m$, so Dini's theorem, see Proposition 1.4.1 of [14], shows that the set $\left\{|S| \mathbf{v}_{n}: n \in \mathbb{N}\right\}$ does not contain any weakly convergent subsequence, which implies that $|S|$ is not weakly compact, so that (1) holds.

For (2) taking $S, T, E, F$ as above, let $E_{1}=E \oplus F$, and define $S_{1}, T_{1}: E_{1} \rightarrow E_{1}$ by $T_{1}(x, y)=(0, T x), S_{1}(x, y)=(0, S x)$, then we have the desired example.

Corollary 2.8. There exist a Dedekind complete Banach lattice $E$ and a pair of positive compact operators $T$ and $S$ on $E$ such that $T \vee S$ and $T \wedge S$ are not weakly compact.

In a similar way we can produce compactly dominated compact operators with a modulus which is neither Dunford-Pettis nor AM-compact.

\section{Vector LATTICES OF WEAKLY COMPACT OPERATORS}

In [5] the idea of a generalized sublattice was introduced to allow discussion of lattice ordered subsets of partially ordered sets which were not themselves lattices, such as the compact operators on $C([0,1])$ (which form a lattice) as a subset of $\mathcal{L}^{r}(C([0,1]))$ (which is not a lattice). There we said that if $(\mathcal{J}, \leq)$ is a partially ordered vector space and $\mathcal{I}$ a subspace of $\mathcal{J}$, then $\mathcal{I}$ is a generalized sublattice of $\mathcal{J}$ if $(\mathcal{I}, \leq)$ is a lattice and for each $x, y \in \mathcal{I}$ the supremum of $x$ and $y$ calculated in $\mathcal{I}$ is also their supremum in $\mathcal{J}$. Recall also that a subset $A$ of a partially ordered set $X$ is order convex if $x \in X, a, b \in A$ and $a \leq x \leq b$ together imply that $x \in A$.

Let $\mathcal{W}(E, F)$ denote the space of all weakly compact operators from a Banach lattice $E$ into a Banach lattice $F$ and let $\mathcal{W}^{r}(E, F)$ be the subspace of $\mathcal{W}(E, F)$ generated by all positive weakly compact operators from $E$ into $F$. It follows from Theorem 2.3 above that $\mathcal{W}(E, F)$ is a generalized sublattice of $\mathcal{L}^{r}(E, F)$ if $E$ is an AL-space and $F$ has property (W1). Moreover $\mathcal{W}(E, F)$ is then a Banach lattice under the operator norm. The next result shows that this happens only for such $F$. 
Theorem 3.1. If $E$ is an infinite dimensional $A L$-space, then the following conditions on a Banach lattice $F$ are equivalent:

(1) F has property (W1).

(2) $\mathcal{W}(E, F)$ is a generalized sublattice of $\mathcal{L}^{r}(E, F)$.

(3) $\mathcal{W}(E, F)$ is a vector lattice.

Proof. Clearly $(1) \Rightarrow(2) \Rightarrow(3)$. Assume that (3) holds and suppose that $E=L_{1}(\mu)$. Since $E$ is infinite dimensional, we may find disjoint measurable sets $A_{1}, A_{2}, A_{3}, \ldots$ $0<\mu\left(A_{i}\right)<\infty$ for each $i \in \mathbb{N}$. Let $A=\bigcup_{i=1}^{\infty} A_{i}$ and define $P, P_{i}: E \rightarrow E$ by $P f=f \chi_{A}, P_{i} f=f \chi_{A_{i}}$ for all $f \in E$. Clearly $P_{i} P_{j}=P_{i}\left(P-P_{i}\right)=\left(P-P_{i}\right) P_{i}=0$ if $i \neq j$, and $P f=\sum_{i=1}^{\infty} P_{i} f$ for all $f \in E$.

If $F$ does not have property (W1), then there exists a weakly convergent sequence $y_{n} \in F$ such that $\left\{\left|y_{n}\right|\right\}$ does not contain any weakly convergent subsequence. Define $S: E=L_{1}(\mu) \rightarrow F$ by

$$
S f=\sum_{n=1}^{\infty}\left(\int_{A_{n}} f d t\right) y_{n}
$$

for all $f \in E$. Since

$$
\sum_{i=1}^{n}\left|\int_{A_{i}} f d t\right| \leq \sum_{i=1}^{n} \int_{A_{i}}|f| d t \leq \int_{A}|f| d t \leq\|f\|
$$

for each $f \in E$, the series is norm convergent. Also

$$
S \operatorname{ball}(E) \subset\left\{\sum_{n=1}^{\infty} \lambda_{n} y_{n}: \sum_{n=1}^{\infty}\left|\lambda_{n}\right| \leq 1\right\}
$$

is relatively weakly compact by Corollary 10.16 of [9], and hence $S$ is weakly compact, i.e. $S \in \mathcal{W}(E, F)$. By (3) the supremum $U$ of $S$ and $-S$ exists in $\mathcal{W}(E, F)$. We claim that

$$
U f=\sum_{n=1}^{\infty}\left(\int_{A_{n}} f d t\right)\left|y_{n}\right|
$$

for each $f \in E$. Indeed, note that $U P \geq \pm S P= \pm S$ and that $U P \in \mathcal{W}(E, F)$ so that $U P \geq U$. Since we certainly have $U P \leq U$, we have $U P=U$. Since $S P_{i} f=\left(\int_{A_{i}} f d t\right) y_{i}$, the modulus $\left|S P_{i}\right|$ (calculated in $\left.\mathcal{L}^{r}(E, F)\right)$ of $S P_{i}$ exists and satisfies

$$
\left|S P_{i}\right| f=\left(\int_{A_{i}} f d t\right)\left|y_{i}\right|
$$

for $f \in E$. Clearly $\left|S P_{i}\right| \in \mathcal{W}(E, F)$ so that $U\left(P-P_{i}\right)+\left|S P_{i}\right| \in \mathcal{W}(E, F)$ and

$$
U\left(P-P_{i}\right)+\left|S P_{i}\right| \geq \pm S\left(P-P_{i}\right) \pm S P_{i}= \pm S P= \pm S .
$$

Hence $U\left(P-P_{i}\right)+\left|S P_{i}\right| \geq U$ which, as $U P=U$, implies that $\left|S P_{i}\right| \geq U P_{i}$. As we clearly have $U P_{i} \geq\left|S P_{i}\right|$ we see that $U P_{i}=\left|S P_{i}\right|$, i.e.

$$
U P_{i} f=\left(\int_{A_{i}} f d t\right)\left|y_{i}\right|
$$

for all $i \in \mathbb{N}$ and all $f \in E$. Therefore

$$
U f=U P f=U\left(\sum_{i=1}^{\infty} P_{i} f\right)=\sum_{i=1}^{\infty} U P_{i} f=\sum_{i=1}^{\infty}\left(\int_{A_{i}} f d t\right)\left|y_{i}\right|
$$

as claimed. 
Now let $g_{n}=\frac{1}{\mu\left(A_{n}\right)} \chi_{A_{n}} \in E$ so that $\left\|g_{n}\right\|_{1}=1$ and $U g_{n}=\left|y_{n}\right|$. The weak compactness of $U$ implies that $\left\{\left|y_{n}\right|: n \in \mathbb{N}\right\}$ contains a weakly convergent subsequence, which is a contradiction, so that $(3) \Rightarrow(1)$ holds.

Combining this with Theorem 17.10 of [9], we have

Corollary 3.2. For a Banach lattice $F$ the following assertions are equivalent

(1) F has the property (W1) and an order continuous norm.

(2) For each $A L$-space $E, \mathcal{W}(E, F)$ is an order convex generalized sublattice of $\mathcal{L}^{r}(E, F)$.

(3) For some infinite dimensional $A L$-space $E, \mathcal{W}(E, F)$ is a lattice and an order convex subset of $\mathcal{L}^{r}(E, F)$.

The next result is the dual version.

Theorem 3.3. Let $F$ be an infinite dimensional Dedekind complete AM-space with unit, E a Banach lattice, then the following assertions are equivalent:

(1) $E^{\prime}$ has the property (W1).

(2) $\mathcal{W}(E, F)$ is a sublattice of $\mathcal{L}^{r}(E, F)$.

(3) $\mathcal{W}(E, F)$ is a vector lattice.

Proof. Clearly (1) $\Rightarrow(2) \Rightarrow(3)$. If (3) holds but (1) is false then there exist $x_{n}^{\prime} \in E$ with $x_{n}^{\prime} \rightarrow x^{\prime} \in E$ weakly as $n \rightarrow \infty$, but $\left\{\left|x_{n}^{\prime}\right|: n \in \mathbb{N}\right\}$ not containing any weakly convergent subsequence.

We may assume that $F=C(X)$ for some Stonean $X$. Since $F$ is infinite dimensional there exists a sequence of disjoint clopen subsets $A_{n}$ of $X$. Define $Q_{n}: F \rightarrow F$ by $Q_{n} f=f \chi_{A_{n}}$ then $0 \leq Q_{n} \leq I_{F}, Q_{m} Q_{n}=Q_{m}\left(I_{F}-Q_{m}\right)=\left(I_{F}-Q_{m}\right) Q_{m}=0$ if $m \neq n$.

Define $S: E \rightarrow F$ by $S x=x^{\prime}(x) \chi_{X}+\sum_{n=1}^{\infty} y_{n}^{\prime}(x) \chi_{A_{n}}$ for $x \in E$, where $y_{n}^{\prime}=x_{n}^{\prime}-x^{\prime}$ which converges weakly to $0 \in E^{\prime}$ as $n \rightarrow \infty$. Define also $T: E \rightarrow F$ by $T x=\sum_{n=1}^{\infty} y_{n}^{\prime}(x) \chi_{A_{n}}$, then it is easy to verify that

$$
T^{\prime} y^{\prime}=\sum_{n=1}^{\infty} y^{\prime}\left(\chi_{A_{n}}\right) y_{n}^{\prime}
$$

for each $y^{\prime} \in F^{\prime}$, and that for each $m \in \mathbb{N}$

$$
\sum_{n=1}^{m}\left|y^{\prime}\left(\chi_{A_{n}}\right)\right| \leq \sum_{i=n}^{m}\left|y^{\prime}\right|\left(\chi_{A_{n}}\right) \leq\left|y^{\prime}\right|\left(\chi_{X}\right)=\left\|y^{\prime}\right\|
$$

so that $\left(y^{\prime}\left(\chi_{A_{n}}\right)\right) \in \ell_{1}$ and $T^{\prime} \operatorname{ball}\left(F^{\prime}\right) \subset\left\{\sum_{n=1}^{\infty} \lambda_{n} y_{n}^{\prime}: \sum_{n=1}^{\infty}\left|\lambda_{n}\right| \leq 1\right\}$ which implies that $T^{\prime}$ is weakly compact by Corollary 10.16 of [9]. Hence $T$ is weakly compact by Gantmacher's theorem, i.e $T \in \mathcal{W}(E, F)$ and hence $S \in \mathcal{W}(E, F)$.

From (3) the supremum $U$ of $S$ and $-S$ exists in $\mathcal{W}(E, F)$, and we claim that $Q_{n} U x=\left|x_{n}^{\prime}\right|(x) \chi_{A_{n}}$. To this end we first note that $Q_{n} U \geq \pm Q_{n} S$ so $Q_{n} U \geq\left|Q_{n} S\right|$, where $\left|Q_{n} S\right|$ is the modulus of $\left|Q_{n} S\right|$ in $\mathcal{L}^{r}(E, F)$ which is given by the formula $\left|Q_{n} S\right| x=\left|x_{i}^{\prime}\right|(x) \chi_{A_{i}}$, so that $\left|Q_{n} S\right| \in \mathcal{W}(E, F)$ and $\left(I_{F}-Q_{n}\right) U+\left|Q_{n} S\right| \in \mathcal{W}(E, F)$. From the inequality $\left(I_{F}-Q_{n}\right) U+\left|Q_{n} S\right| \geq \pm S$ we see that $\left(I_{F}-Q_{n}\right) U+\left|Q_{n} S\right| \geq U$ and

$$
\left|Q_{n} S\right| \geq Q_{n}\left|Q_{n} S\right|=Q_{n}\left[\left(I_{F}-Q_{n}\right) U+\left|Q_{n} S\right|\right] \geq Q_{n} U .
$$

Thus $Q_{n} U=\left|Q_{n} S\right|$, so that $Q_{n} U x=\left|x_{n}^{\prime}\right|(x) \chi_{A_{n}}$ for each $n \in \mathbb{N}, x \in E$. 
Choose $t_{n} \in A_{n}$ and define $\delta_{n}(f)=f\left(t_{n}\right)$ for $f \in F$, then $\delta_{n} \in F_{+}^{\prime}$ with $\left\|\delta_{n}\right\|=1$. Clearly

$$
Q_{n}^{\prime} \delta_{m}= \begin{cases}\delta_{n}, & m=n, \\ 0, & m \neq n,\end{cases}
$$

for all $m, n \in \mathbb{N}$. For each $x \in E$,

$$
\begin{aligned}
\left(U^{\prime} \delta_{n}\right)(x) & =\delta_{n}(U x)=Q_{n}^{\prime} \delta_{n}(U x) \\
& =\delta_{n}\left(Q_{n} U x\right)=\left|x_{n}^{\prime}\right|(x)
\end{aligned}
$$

and it follows that $U^{\prime} \delta_{n}=\left|x_{n}^{\prime}\right|$ for $n \in \mathbb{N}$. Now the assumed weak compactness of $U$ and hence of $U^{\prime}$ implies that $\left\{\left|x_{n}^{\prime}\right|: n \in \mathbb{N}\right\}$ contains a weakly convergent subsequence. This is a contradiction so that $(3) \Rightarrow(1)$ holds.

Combining this with Theorem 17.10 of [9] we have

Corollary 3.4. For a Banach lattice $E$ the following assertions are equivalent:

(1) $E^{\prime}$ has an order continuous norm (and is hence a KB-space).

(2) For each Dedekind complete AM-space $F$ with unit, $\mathcal{W}(E, F)$ is an ideal of $\mathcal{L}^{r}(E, F)$.

(3) For some infinite dimensional Dedekind complete $A M$-space $F, \mathcal{W}(E, F)$ is an ideal of $\mathcal{L}^{r}(E, F)$.

It is well-known that $\mathcal{W}(E, F) \not \subset \mathcal{L}^{r}(E, F)$ in general. As in the case of compact operators we define the subspace $\mathcal{W}^{r}(E, F)$ to be the linear span of the space of all positive weakly compact operators from $E$ into $F$. The operators constructed in the course of the proof of Theorem 2.7 (a) show, given the atomic nature of the domain, that even $\mathcal{W}^{r}(E, F)$ need not be a vector lattice. When we consider Dedekind completeness of $\mathcal{W}^{r}(E, F)$ we obtain results similar to those that hold for spaces of compact operator; see [17] for details in that case.

Theorem 3.5. For Banach lattices $E$ and $F$ the following assertions are equivalent:

(1) One of the following two conditions holds:

(a) $E^{\prime}$ has an order continuous norm and $F$ is Dedekind complete.

(b) $F$ has an order continuous norm.

(2) $F$ is Dedekind complete and if $0 \leq S \leq T \in \mathcal{W}(E, F)$, then $S \in \mathcal{W}(E, F)$.

(3) $\mathcal{W}^{r}(E, F)$ is a Dedekind complete vector lattice.

(4) Any increasing order bounded set in $\mathcal{W}^{r}(E, F)$ has a supremum.

Proof. Clearly $(1) \Rightarrow(2) \Rightarrow(3) \Rightarrow(4)$. It is routine that (4) implies that $F$ is Dedekind complete. To see that $(4) \Rightarrow(1)$ it suffices to show that either $E^{\prime}$ or $F$ has an order continuous norm. Otherwise we may assume that $\ell_{1}$ and $\ell_{\infty}$ are closed sublattices of $E$ and $F$ respectively. According to Proposition 2.3.11 of [14] there exists a positive projection $P$ from $E$ onto $\ell_{1}$. Let $\phi_{n}, \phi \in\left(\ell_{1}\right)^{\prime}=\ell_{\infty}$ with

$$
\phi_{n}\left(\left(\lambda_{k}\right)\right)=\lambda_{n}
$$

and

$$
\phi\left(\left(\lambda_{k}\right)\right)=\sum_{k=1}^{\infty} \lambda_{k} .
$$


Define $T_{n}, U_{n}: \ell_{1} \rightarrow \ell_{\infty} \subset F$ by

$$
T_{n}(\lambda)=\sum_{k=1}^{n} \phi_{k}(\lambda) \bar{e}_{k}
$$

(where $\bar{e}_{n}=e_{1}+e_{2}+\cdots+e_{n} \in \ell_{\infty}$ ) and

$$
U_{n}(\lambda)=\phi(\lambda) \mathbf{1}+\sum_{k=1}^{n} \phi_{k}(\lambda)\left(\bar{e}_{k}-\mathbf{1}\right)
$$

for all $\lambda \in \ell_{1}$. Clearly $0 \leq T_{n} \uparrow$ and $0 \leq U_{n} \downarrow$. In a similar way to that used in the proof of Theorem 2.1 of [17], we further have $T_{n} \leq U_{m}$ for all $n, m \in \mathbb{N}$. Thus $0 \leq T_{n} P \uparrow, 0 \leq U_{n} P \downarrow$ and $T_{n} P \leq U_{m} P$ for all $n, m \in \mathbb{N}$. From (4) the supremum $S$ of $\left\{T_{n} P\right\}$ exists in $\mathcal{W}^{r}(E, F)$. Clearly $T_{n} P \leq S \leq U_{m} P$ for all $m, n \in \mathbb{N}$ and it follows that

$$
\bar{e}_{k}=T_{k} P e_{k} \leq S e_{k} \leq U_{k} P e_{k}=\bar{e}_{k} .
$$

But $\left\{\bar{e}_{k}: k \in \mathbb{N}\right\}$ does not contain any subsequence which is weakly convergent in $\ell_{\infty}$ and hence none weakly convergent in $F$, which contradicts the weak compactness of $S$.

It is worth pointing out that if $\mathcal{W}^{r}(E, F)$ is a Dedekind $\sigma$-complete vector lattice, then $F$ must be Dedekind $\sigma$-complete and either $E^{\prime}$ or $F$ have an order continuous norm. However the converse is false.

If we let $E$ or $F$ range over all Banach lattices we have the following two results.

Theorem 3.6. For a Banach lattice $F$ the following assertions are equivalent:

(1) $F$ has an order continuous norm.

(2) For every Banach lattice $E, \mathcal{W}^{r}(E, F)$ is a Dedekind complete vector lattice.

(3) $\mathcal{W}^{r}\left(\ell_{1}\left(L_{2}[0,1]\right), F\right)$ is a vector lattice.

Proof. Clearly $(1) \Rightarrow(2) \Rightarrow(3)$. The proof of $(3) \Rightarrow(1)$ is carried out in two steps.

As a first step we show that $F$ is Dedekind $\sigma$-complete. By Corollary 2.1.6 of [14] it suffices to show that $\sup \left\{y_{n}: n \in \mathbb{N}\right\}$ exists for each order bounded disjoint sequence $y_{n} \in F_{+}$. Fix such a sequence, bounded above by $y \in F_{+}$and define $S, T: E \rightarrow F$ by

$$
S\left(f_{k}\right)=\sum_{n=1}^{\infty}\left(\int_{0}^{1} f_{1} r_{n} d t\right) y_{n}
$$

and

$$
T\left(f_{k}\right)=\left(\int_{0}^{1} f_{1} d t\right) y
$$

for $\left(f_{k}\right) \in E=\ell_{1}\left(L_{2}[0,1]\right)$, where $r_{n}$ is the $n$ 'th Rademacher function on [0,1]. Let $P: E \rightarrow L_{2}[0,1]$ be defined by $P\left(f_{k}\right)=f_{1}$ for $\left(f_{k}\right) \in E$ and $H: L_{2}[0,1] \rightarrow F$ by $H f=\sum_{n=1}^{\infty}\left(\int_{0}^{1} f r_{n} d t\right) y_{n}$, so that $S=H P$ is weakly compact and $\pm S \leq T$. Thus $S, T \in \mathcal{W}^{r}(E, F)$ and by (3) the supremum $U$ of $S$ and $-S$ exists in $\mathcal{W}^{r}(E, F)$ and $\pm S \leq U \leq T$.

For each $0 \leq f \in L_{2}[0,1]$ let $\bar{f}=(f, 0,0, \ldots) \in E$. Using the fact that $(U \pm S)\left(\bar{\chi}_{[0,1]} \pm \bar{r}_{n}\right) \geq 0$ we see that $U \bar{\chi}_{[0,1]} \geq S \bar{r}_{n}=y_{n}$ for all $n \in \mathbb{N}$. Thus

$$
0 \leq y_{n} \leq U \bar{\chi}_{[0,1]} \leq T \bar{\chi}_{[0,1]}=y
$$


for all $n \in \mathbb{N}$. This argument holds for any choice of upper bound $y$ for the set $\left\{y_{n}: n \in \mathbb{N}\right\}$, so that $U \bar{\chi}_{[0,1]}=\sup \left\{y_{n}: n \in \mathbb{N}\right\}$.

We now show that the norm on $F$ is order continuous. Let $y_{n} \in F_{+}$be disjoint with supremum $y$. By Theorem 12.13 of [9], it suffices to prove that $\left\|y_{n}\right\| \rightarrow 0$. Define $S, T: E \rightarrow F$ by $S g=\sum_{n=1}^{\infty} g\left(x_{n}\right) y_{n}$ and $T g=g\left(x_{0}\right) y$ for $g \in E$, where

$$
x_{n}=(\overbrace{r_{n}, \ldots, r_{n}}^{n}, 0, \ldots) \in c_{0}\left(L_{2}[0,1]\right) \subset \ell_{\infty}\left(L_{2}[0,1]\right)=E^{\prime}
$$

and $x_{0}=\left(\chi_{[0,1]}, \chi_{[0,1]}, \ldots\right) \in E^{\prime}$. It is not difficult to check that $x_{n} \rightarrow 0$ weakly in $c_{0}\left(L_{2}[0,1]\right)$ so that the series of disjoint terms is norm convergent. For each $y^{\prime} \in F^{\prime}$ we have

$$
\sum_{i=1}^{n}\left|y^{\prime}\left(y_{n}\right)\right| \leq \sum_{i=1}^{n}\left|y^{\prime}\right|\left(y_{n}\right) \leq\left|y^{\prime}\right|(y) \leq\|y\|\left\|y^{\prime}\right\|
$$

for all $n \in \mathbb{N}$, so that $\left(y^{\prime}\left(y_{n}\right)\right) \in \ell_{1}$. Note also that if $g \in E$, then

$$
S^{\prime} y^{\prime}(g)=y^{\prime}(S g)=\sum_{n=1}^{\infty} x_{n}(g) y^{\prime}\left(y_{n}\right)=\left(\sum_{n=1}^{\infty} y^{\prime}\left(y_{n}\right) x_{n}\right)(g)
$$

so that

$$
\begin{aligned}
S^{\prime} \operatorname{ball}\left(F^{\prime}\right) & \subset\left\{\sum_{n=1}^{\infty} \lambda_{n} x_{n}: \sum_{n=1}^{\infty}\left|\lambda_{n}\right| \leq\|y\|\right\} \\
& =\|y\|\left\{\sum_{n=1}^{\infty} \lambda_{n} x_{n}: \sum_{n=1}^{\infty}\left|\lambda_{n}\right| \leq 1\right\}
\end{aligned}
$$

which is relatively weakly compact by Corollary 10.16 of [9]. So $S^{\prime}$, and hence $S$, are weakly compact. Clearly $\pm S \leq T$ so $S, T \in \mathcal{W}^{r}(E, F)$. By (3) the supremum $U$ of $S$ and $-S$ exists in $\mathcal{W}^{r}(E, F)$. Moreover $U Q_{k}\left(f_{n}\right)=\left(\int_{0}^{1} f_{k} d t\right)\left(y-\sum_{i=1}^{k-1} y_{i}\right)$, where $Q_{k}: E \rightarrow E$ maps $\left(f_{n}\right) \in E$ to the sequence with $k$ 'th entry equal to $f_{k}$ and all others zero. Now, since $U Q_{k} \geq \pm S Q_{k}$ and $S Q_{k}\left(f_{n}\right)=\sum_{i=k}^{\infty}\left(\int_{0}^{1} f_{k} r_{k} d t\right) y_{i}$, it is easy to verify that the modulus $\left|S Q_{k}\right|$ of $S Q_{k}$ exists in $\mathcal{L}^{r}(E, F)$ and that $\left|S Q_{k}\right|\left(f_{n}\right)=\left(\int_{0}^{1} f_{k} d t\right)\left(y-\sum_{i=1}^{k-1} y_{i}\right)$. Thus $U Q_{k} \geq\left|S Q_{k}\right|$ which is weakly compact. As $U\left(I_{E}-Q_{k}\right)+\left|S Q_{k}\right| \geq \pm S$ it follows that $U\left(I_{E}-Q_{k}\right)+\left|S Q_{k}\right| \geq U$ and hence

$$
\left|S Q_{k}\right| \geq\left|S Q_{k}\right| Q_{k}=\left(U\left(I_{E}-Q_{k}\right)+\left|S Q_{k}\right|\right) Q_{k} \geq U Q_{k}
$$

which implies that $U Q_{k}=\left|S Q_{k}\right|$ so that $U Q_{k}\left(f_{n}\right)=\left(\int_{0}^{1} f_{k} d t\right)\left(y-\sum_{i=1}^{k-1} y_{i}\right)$. In particular $U h_{n}=y-\sum_{i=1}^{n-1} y_{i} \downarrow$, where $h_{n} \in E$ denotes the sequence with $n$ 'th entry equal to $\chi_{[0,1]}$ and all others zero. It follows from the weak compactness of $U$ that $\left(y-\sum_{i=1}^{k-1} y_{n}\right)$ norm converges to 0 (noting that $0=\inf \left\{y-\sum_{i=1}^{k-1} y_{i}: k \in \mathbb{N}\right\}$ ) which implies that $\left\|y_{n}\right\| \rightarrow 0$ as $n \rightarrow \infty$. Therefore $F$ has an order continuous norm as claimed.

In the similar way, we have the following dual version.

Theorem 3.7. For a Banach lattice $E$ the following assertions are equivalent:

(1) $E^{\prime}$ has an order continuous norm (and is hence a KB-space).

(2) For each Dedekind complete Banach lattice $F, \mathcal{W}^{r}(E, F)$ is a Dedekind complete vector lattice.

(3) $\mathcal{W}^{r}\left(E, \ell_{\infty}\left(L_{2}[0,1]\right)\right)$ is a vector lattice. 
If $\mathcal{D}^{r}(E, F)$ denotes the subspace of $\mathcal{L}^{r}(E, F)$ generated by all positive DunfordPettis operators from $E$ into $F$, then we have:

Theorem 3.8. For Banach lattices $E$ and $F$ the following assertions are equivalent:

(1) One of the following two conditions holds.

(a) $F$ is Dedekind complete and the lattice operations in $E$ are weakly sequentially continuous.

(b) F has an order continuous norm.

(2) $F$ is Dedekind complete and if $0 \leq S \leq T \in \mathcal{D}^{r}(E, F)$, then $S \in \mathcal{D}^{r}(E, F)$.

(3) $\mathcal{D}^{r}(E, F)$ is a Dedekind complete vector lattice.

(4) Any increasing order bounded set in $\mathcal{D}^{r}(E, F)$ has a supremum.

Proof. Clearly $(1) \Rightarrow(2) \Rightarrow(3) \Rightarrow(4)$. To see that $(4) \Rightarrow(1)$ it suffices to show that either the lattice operations of $F$ are weakly sequentially continuous or $F$ has an order continuous norm, as (4) certainly implies that $F$ is Dedekind complete.

If (a) fails, then there exist $x_{n} \in E, x^{\prime} \in E_{+}^{\prime}$ and $0<\epsilon \in \mathbb{R}$ such that $x_{n} \rightarrow 0$ weakly in $E$ as $n \rightarrow \infty$ and $x^{\prime}\left(\left|x_{n}\right|\right) \geq 2 \epsilon>0$ for all $n \in \mathbb{N}$. If (b) also fails, then there is a disjoint sequence $y_{n} \in F_{+}$with supremum $y$ and $\left\|y_{n}\right\| \geq 1$. It follows from Lemma 1.4.4 of [14] that there exists $x_{n}^{\prime} \in\left[0, x^{\prime}\right]$ such that $\left|x_{n}^{\prime}\left(x_{n}\right)\right| \geq \epsilon>0$ for each $n \in \mathbb{N}$.

Define $S_{n}, T: E \rightarrow F$ by $S_{n} x=\sum_{i=1}^{n} x_{n}^{\prime}(x) y_{n}$ and $T x=x^{\prime}(x) y$. Then $0 \leq$ $S_{n} \uparrow \leq T$ and $S_{n}, T \in \mathcal{D}^{r}(E, F)$. By (4) the supremum $S$ of $\left\{S_{n}\right\}$ exists in $\mathcal{D}^{r}(E, F)$.

Let $Q_{n}$ be the band projection of $F$ onto the band $B_{n}$ generated by $y_{n}$, then the disjointness of $y_{n}$ implies that $Q_{n} S_{m}=Q_{n} S_{n}$ for all $m \geq n$, and $Q_{n} S_{n} \in \mathcal{D}^{r}(E, F)$ so that $\left(I_{F}-Q_{n}\right) S+Q_{n} S_{n} \in \mathcal{D}^{r}(E, F)$ and we note that $\left(I_{F}-Q_{n}\right) S+Q_{n} S_{n} \geq S_{m}$ for all $m$. It follows that $\left(I_{F}-Q_{n}\right) S+Q_{n} S_{n} \geq S$ which implies that $Q_{n} S_{n} \geq Q_{n} S$ for all $n$. Now for each $x \in E$

$$
|S x| \geq Q_{n}|S x|=\left|Q_{n} S x\right|=\left|Q_{n} S_{n} x\right|=\left|x_{n}^{\prime}(x)\right| y_{n} .
$$

In particular

$$
\left|S x_{n}\right| \geq\left|x_{n}^{\prime}\left(x_{n}\right)\right| y_{n} \geq \epsilon y_{n}
$$

so that $\left\|S x_{n}\right\| \geq \epsilon\left\|y_{n}\right\| \geq \epsilon>0$ for all $n$. This means that $S \notin \mathcal{D}^{r}(E, F)$ which is a contradiction.

\section{Comments}

(1) The following question was asked in [3] and modified in [4]: Does there exist a pair of Dedekind complete Banach lattices $E$ and $F$ such that at least one of $E$ and $F$ is reflexive and $\mathcal{K}(E, F) \subset \mathcal{L}^{r}(E, F)$ but $\mathcal{L}^{r}(E, F) \neq \mathcal{L}(E, F)$ ? This question is answered positively by taking $E=L_{2}[0,1]$ and $F=c_{0}$, so that $E$ is reflexive and $\mathcal{K}(E, F) \subset \mathcal{L}^{r}(E, F)$ but $\mathcal{L}^{r}(E, F) \neq \mathcal{L}(E, F)$.

(2) The following question was also asked in [3] for the case of weak compactness: Does there exist a pair of Dedekind complete Banach lattices $E$ and $F$ such that $\mathcal{W}(E, F) \subset \mathcal{L}^{r}(E, F)$ and $\mathcal{L}^{r}(E, F) \neq \mathcal{L}(E, F)$ ? Again we obtain a positive answer by taking $E=L_{1}[0,1]$ and $F=c_{0}$. Theorem 3.1 above implies that $\mathcal{W}(E, F)$ is a sublattice of $\mathcal{L}^{r}(E, F)$ but $\mathcal{L}^{r}(E, F) \neq \mathcal{L}(E, F)$. 


\section{REFERENCES}

[1] Y. A. Abramovich, Weakly compact sets in topological $K$-spaces, Teor. Funkcii Funkcional. Anal. i Prilozen. (Kharkov) 15 (1972), 27-35. MR 46:5982

[2] Y. A. Abramovich, E.D. Posizelsky and L.P. Janovsky, On some parameters associated with normed lattices and on series characterisation of M-spaces, Studia Math. 63 (1978), 1-8. MR 80e: 46014

[3] Y. A. Abramovich, When each regular operator is continuous, Functional Analysis, Optimization and Mathematical Economics, Oxford University Press, New York, 1990, pp. 133-140. MR 91m:47051

[4] Y. A. Abramovich and A. W. Wickstead, A compact regular operator without modulus, Proc. Amer. Math. Soc. 116 (1992), 721-726. MR 93a:47038

[5] Y. A. Abramovich and A. W. Wickstead, Recent results on the order structure of compact operators, Irish Math. Soc. Bulletin 32 (1994), 32-45. MR 95h:47050

[6] Y. A. Abramovich and A. W. Wickstead, Solutions of several problems in the theory of compact positive operators, Proc. Amer. Math. Soc. 123 (1995), 3021-3026. MR 95m:47059

[7] Y. A. Abramovich, Z. L. Chen and A.W. Wickstead, Regular-norm balls can be closed in the strong operator topology, Positivity 1 (1997), 75-96.

[8] C. D. Aliprantis and O. Burkinshaw, On weakly compact operators on Banach lattices, Proc. Amer. Math. Soc. 83 (1981), 573-578. MR 82j:47057

[9] C. D. Aliprantis and O. Burkinshaw, Positive Operators, Academic Press, New York \& London, 1985. MR 87h:47086

[10] Z. L. Chen and A. W. Wickstead, Relative weak compactness of solid hulls in Banach lattices (submitted).

[11] J. Diestel, Sequences and series in Banach spaces, Springer-Verlag, New York, Berlin, Heidelberg, Tokyo, 1984. MR 85i:46020

[12] G. Groenewegen, On spaces of Banach lattice valued functions and measures (Thesis), Nijmegen University, Netherlands, 1982.

[13] G. Groenewegen and A. van Rooij, The modulus of a weakly compact operator, Math. Z. 195 (1987), 473-480. MR 88h:47052

[14] P. Meyer-Nieberg, Banach Lattices, Springer-Verlag, Berlin, Heidelberg, New York, 1991. MR 93f: 46025

[15] C. P. Niculescu, Weak compactness in Banach lattices, J. Operator Theory 6 (1981), 217-231. MR 83d: 47044

[16] K. D. Schmidt, On the modulus of weakly compact operators and strongly additive vector measures, Proc. Amer. Math. Soc. 102 (1988), 862-866. MR 89e:47056

[17] A. W. Wickstead, Dedekind completeness of some lattices of compact operators, Bull. Pol. Acad. Sci. 43 (1995), 297-304. MR 97k:47032

Department of Applied Mathematics, Southwest Jiaotong University, Chengdu Sichuan 610031, People's Republic of China

Department of Pure Mathematics, The Queen's University of Belfast, Belfast BT7 1 NN, NorThern IRELAND

E-mail address: A. Wickstead@qub.ac.uk 